\title{
Evaluación participativa de los impactos sociales del turismo residencial y meta- análisis de resultados: un caso de estudio en Pipa (Rio Grande do Norte, Brasil)
}

\section{Participatory social impact assessment of residential tourism and meta-analysis of results: a case study in Pipa (Rio Grande do Norte, Brazil)}

\author{
Antonio Aledo (ALEDO, A.) \\ Hugo García-Andreu (GARCIA-ANDREU, H.) ${ }^{* *}$ \\ Guadalupe Ortiz (ORTIZ, G.) ${ }^{* * *}$
}

RESUMEN - Este artículo presenta una propuesta metodológica para la evaluación participativa de impactos sociales. Se ejemplifica mediante la descripción del proceso y resultados de una investigación realizada en la comunidad turística de Pipa (Rio Grande do Norte, Brasil) en la que se desarrolló un proceso de participación orientado a discutir el modelo de turismo residencial implantado en este territorio mediante el identificación y evaluación de sus impactos sociales. La novedad de esta propuesta reside en que se añade, a los beneficios y utilidades de la evaluación participativa de impactos sociales, un meta-análisis realizado sobre los resultados del proceso de participación. Este metaanálisis hace uso de las herramientas informáticas propias del Análisis de Redes Sociales aplicadas al estudio de los mapas causa-efecto elaborados por los participantes. Los resultados de este análisis cuantitativo se completan e interpretan con la información obtenida a través de entrevistas en profundidad y revisión documental, permitiendo: 1) identificar las causas últimas de los impactos sociales derivados del turismo residencial a escala local y 2) una mejor comprensión de la complejidad causal de estos impactos. El meta-análisis ha identificado que la primacía de los intereses de las empresas inmobiliarias internacionales sobre el interés general local se sustenta sobre su capacidad ilusoria de controlar la demanda mediante agresivas campañas de marketing. Esta información permite la deconstrucción del discurso desarrollista del turismo y posibilita la demarcación de nuevas áreas de acción estratégica orientadas a la maximización del beneficio colectivo.

Palabras clave: Análisis de Redes Sociales; Mapas causales; Evaluación Participativa; Turismo Residencial; Impactos.

ABSTRACT - This paper presents a methodological proposal for the participatory assessment of social impacts. This is illustrated with the description of the process and results of a research developed at the tourism destination of Pipa (Rio Grande do Norte,

\footnotetext{
* Doctorado en Sociología (Universidad de Alicante). Profesor Titular de Sociología, Departamento de Sociología I de la Universidad de Alicante. Dirección: Departamento de Sociología I, Universidad de Alicante. Apdo. 99. 03080. Alicante (España). Teléfono: +34 965909586. E-mail: antonio.aledo@ua.ese

** Doctorado en Sociología (Universidad de Alicante). Profesor en el Departamento de Sociología I de la Universidad de Alicante. Dirección: Departamento de Sociología I, Universidad de Alicante. Apdo. 99. 03080. Alicante (España). Teléfono: +34 965909772. E-mail: hugo.andreu@ua.es

** Doctorado en Sociología (Universidad de Alicante) y Máster en Estrategias y Gestión Ambiental (Universidad de Valencia). Profesora en el Departamento de Sociología I de la Universidad de Alicante. Dirección: Departamento de Sociología I, Universidad de Alicante. Apdo. 99. 03080. Alicante (España). Teléfono: +34 965909772. E-mail: guadalupe.ortiz@ua.es
} 
Brazil). At this destination, a participatory process was conducted with the aim of discussing the model of residential tourism established in the area, through the identification and assessment of its social impacts. The novelty in this proposal lies in the addition to the benefits and usefulness of participatory impact assessment of a metaanalysis of the outcomes of the participatory process. This meta-analysis applies the computer tools used in Social Networks Analysis to the study of the cause-effect maps elaborated by the participants. The results of this quantitative analysis are completed and interpreted with the information obtained through qualitative interviews and documentation, allowing: 1) the identification of the deep causes of the social impacts derived from residential tourism at a local level and 2) a better understanding of the causal complexity of these impacts. The meta-analysis has identified that the primacy of the international real estate companies' interests over the local general interest is sustained on their illusory capacity to control the demand by means of aggressive advertising campaigns. This information permits the deconstruction of the tourism developmentalist discourse and enables the demarcation of new areas for strategic action oriented to the maximization of collective benefits.

Key words: Social Network Analysis; Causal maps; Participatory Assessment; Residential Tourism; Impacts. 


\section{INTRODUCCIÓN ${ }^{1}$}

El turismo residencial - entendido como el modelo de desarrollo local que se estructura en torno a la venta de viviendas secundarias en espacios de especialización turística (O’REILLY, 2007, 2009) - se consolidó en el Mediterráneo occidental durante las tres últimas décadas del pasado siglo (BRAMWELL, 2004; ALEDO, 2008). A comienzos del nuevo siglo, las expectativas generadas tanto por el turismo como, muy especialmente, por la burbuja inmobiliaria global propiciaron su salto atlántico (BLÁZQUEZ, CAÑADA y MURRAY, 2011). Entre el 2002 y el 2007, el turismo residencial experimentó un periodo de corto pero intenso crecimiento en Latinoamérica (HIERNAUX, 2010), crecimiento que la crisis financiera frenó bruscamente en 2008 (NAREDO, 2010; MONTI, 2011). Durante dicho sexenio se construyeron miles de unidades residenciales orientadas fundamentalmente al mercado extranjero aprovechando una serie de ventajas comparativas relacionadas con un paisaje escasamente turistizado, el bajo coste de la tierra y de la mano de obra, el apoyo gubernamental a la inversión extranjera y una gran disponibilidad de capitales financieros internacionales de carácter especulativo en busca de nichos estables de inversión (DANTAS, FERREIRA y LIBRAMENTO, 2010).

En el caso brasileño, la región elegida por el turismo residencial fue el Nordeste. Según el informe de 2005 del PNUD, el Índice de Desarrollo Humano del Nordeste era el más bajo de todas las regiones brasileñas (BANCO CENTRAL DO BRASIL, 2009). El peso de las estructuras coloniales, la dureza ambiental del Sertão y la sucesión de crisis de la industria del azúcar desde finales del siglo XIX explican que el Nordeste sea una de las zonas menos desarrolladas de Brasil y un foco de emigración hacia las metrópolis del sur (DANTAS, 2007). La llegada del turismo a comienzos de la década de los ochenta fue vista como una oportunidad para alterar esa desventajosa posición (MONTI, 2011).

El destino turístico-residencial escogido para esta investigación, Pipa, en el municipio de Tibau do Sul, representa un ejemplo paradigmático de la evolución del

\footnotetext{
${ }^{1}$ Una versión menos desarrollada de este artículo fue presentada en el Colloquium Methodological Challenges in Participation Research, que tuvo lugar en noviembre de 2011 en Córdoba, España, organizado por el IESA-CSIC. Este trabajo es el resultado de la investigación "Causas y consecuencias de la globalización del turismo de segunda residencia: del Mediterráneo español al litoral nordestino brasileño" (CSO2009-14074), financiada por el Ministerio de Ciencia e Innovación de España.
} 
turismo en el nordeste brasileño. Se trata de un distrito que contaba con 11.402 habitantes en 2010 (INSTITUTO BRASILEIRO DE GEOGRAFIA E ESTATÍSTICA, 2013), situado a $85 \mathrm{~km}$ de Natal, la capital del Estado. Antiguamente una villa de pescadores, es hoy el segundo destino turístico de Rio Grande do Norte (PEREIRA, 2012). Hasta finales de los noventa, el modelo turístico dominante en esta localidad fue el hotelero. En una primera etapa, se instalaron pequeñas posadas y, a partir de mediados de los noventa, se establecieron algunas cadenas hoteleras internacionales. Su llegada estuvo acompañada por el esfuerzo del sector público por desarrollar unas infraestructuras que facilitasen el desarrollo turístico (CRUZ, 2000) a través del Programa de Desarrollo del Turismo en el Nordeste, PRODETUR/NE (BANCO DO NORDESTE, 2006 y 2009). Este destino reorientó su modelo a partir de 2002, cuando el litoral nordestino experimentaba un intenso crecimiento inmobiliario como consecuencia de inversiones extranjeras en proyectos de segundas residencias (RIBEIRO, 2009; SILVA y FONSECA, 2010). En toda la costa, la multiplicación de condominios turísticos cerrados destinados a una clientela europea fue posible gracias a, por un lado, una coyuntura económica global favorable a la inversión inmobiliaria y, por otro, a políticas turísticas orientadas a integrar esta región periférica en los circuitos internacionales del turismo (LOLOUM, 2010; MONTI, 2011).

Esta última fase de desarrollo turístico inmobiliario extranjero ha tenido una corta carrera en Pipa al igual que en el resto de la costa nordestina ya que, iniciándose en 2002, finalizó en 2008. Pero, a pesar de su corto ciclo de crecimiento y expansión, este nuevo fenómeno ha generado importantes transformaciones sobre la comunidad local y su entorno. Si el sector hotelero había creado cerca de 5.000 plazas en tres décadas, el sector turístico residencial desarrolló un número equivalente en tan solo un quinquenio (LOLOUM, 2010). El sector turístico residencial ha generado importantes beneficios económicos pero, al mismo tiempo, ha producido cambios de carácter ambiental y sociocultural que son percibidos por la población local como impactos negativos. El modelo extensivo de ocupación del territorio - incluso sobre espacios protegidos -, la competencia con el sector hotelero, el debilitamiento de las actividades económicas tradicionales, sumado a la inversión de los escasos recursos públicos hacia las áreas turísticas, han generado toda una serie de efectos negativos en la sociedad local y el territorio. A todo ello se añade una distribución desigual de los perjuicios y 
beneficios entre los diferentes grupos sociales que componen la comunidad local.

A pesar de que muchos de los impactos negativos en Pipa son evidentes, la ausencia de evaluaciones de impacto social hace que todavía no estén claros los procesos causales que los originan y alimentan. Evaluar correctamente esta cuestión resulta fundamental para desarrollar e implementar medidas correctoras de tales impactos negativos. En esta línea, el presente trabajo centra su atención en la evaluación participativa de impactos sociales del desarrollo turístico en Pipa.

Existe un amplio consenso sobre la pertinencia de incorporar en las evaluaciones de impacto social la participación de aquellos actores sociales afectados de un modo u otro por dichos impactos (BANCO MUNDIAL, 1993; BURDGE, 1995; BECKER et al., 2003; ROBERTS, 2003; VANCLAY, 2003; TAYLOR, BRYAN y GOODRICH, 2004). La unión entre evaluación de impacto social y participación se justifica, por un lado, a partir de un argumento normativo, en tanto que, para asegurar la justicia y legitimidad de los resultados, la pluralidad de intereses y opiniones de los afectados por el proceso de cambio evaluado debe estar presente y participar activamente en el proceso evaluador (VANCLAY, 2003). Y por otro lado, se justifica por un argumento pragmático, en tanto que la participación facilita la identificación de estrategias de actuación frente a los impactos negativos del proceso de cambio más adaptadas a la realidad social. Se garantiza de esta manera una correcta contextualización local de los resultados de la evaluación, el aprendizaje social, la identificación y prevención de potenciales conflictos entre actores sociales, así como una mayor comprensión, aceptación y sentimiento de apropiación de los resultados por parte de los implicados (BURDGE y VANCLAY, 1996; BECKER et al., 2003; BECKER y VANCLAY, 2003; VANCLAY, 2003; ROBERTS, 2003).

El turismo como industria global tiene la capacidad de afectar y transformar de forma profunda los sistemas sociales, especialmente en regiones en desarrollo (MATHIESON y WALL, 1982; CRANDALL, 1987; MATTEUCCI, LUNDDURLACHER y BEYER, 2008). Los impactos del turismo afectan tanto al medio ambiente como al entorno social (GREENPEACE, 2009), generando cambios profundos que pueden modificar las estructuras locales (FONSECA y ADEMIR, 2005; LEON, 2007). Los efectos positivos y negativos se distribuyen de forma desigual entre los diferentes grupos que participan de la actividad turística (BLÁZQUEZ y CAÑADA, 
2011). Todo ello justifica la necesidad de incluir a la ciudadanía y actores implicados en los procesos evaluación de los impactos del turismo, si lo que se persigue es hacer más justos, legítimos y eficaces los procesos de toma de decisiones. De este modo, cuando se habla de participación en este trabajo, se está haciendo referencia a procesos reflexivos en los que ciudadanos afectados o interesados por las cuestiones tratadas exponen, discuten y elaboran sus opiniones, intereses y propuestas para su incorporación en programas de carácter técnico y/o político (ORTIZ, 2009). No son pocos los autores que han señalado la necesidad de abrir los procesos de planificación y gestión turística a otros actores sociales (MURPHY, 1985; AP 1992; JAMAL y GETZ, 1995; BROHMAN, 1996; TOSUN y TIMOTHY, 2003; ORTIZ et al., 2007; ORTIZ, 2009; GARCÍA-ANDREU, ALEDO y ORTIZ, 2010). En concreto, la relevancia de la evaluación participativa de los impactos sociales del turismo radica en su capacidad para 1) identificar los grupos que son excluidos de los beneficios de la actividad turística y sobre quienes repercuten en mayor medida los costes; 2) identificar los procesos que originan esa exclusión y 3) generar información de calidad para la formulación de propuestas y acciones orientadas a disminuir esa desigual distribución de los recursos e impactos.

Usualmente los diagnósticos de impacto social del turismo han adolecido de falta de integración y superficialidad al estar fundamentalmente preocupados por la cualificación sectorial del producto turístico de cara a no perder competitividad en el mercado (GARCÍA-ANDREU, 2008). Este tipo de diagnósticos han tenido como consecuencia que la mayor parte de las propuestas de actuación y mejora tuvieran un carácter eminentemente técnico. En esta línea, se ha señalado que un parte importante de los problemas que padecen los municipios turísticos son resultado de una rápida y errónea planificación urbanística y turística desarrollada para atender las exigencias de una demanda en constante y rápido crecimiento (MUNRES, 1995; NAVALÓN, 1995; VERA, 1997; MAZÓN, 2006). Sin embargo, estos diagnósticos obvian que la errónea planificación es la manifestación de causas más profundas que conectan con las estructuras de poder locales. Si no se adopta una aproximación integrada y estructural en el análisis del modelo de desarrollo turístico-residencial resulta complicado identificar las causas de fondo que condicionan la orientación de las herramientas de planificación y su correcta aplicación. 
De este modo, y teniendo como objetivo la identificación de las causas últimas de los impactos sociales del turismo, esta investigación ofrece una propuesta metodológica para el análisis de la información producida durante un proceso participativo. Se plantea el uso de las herramientas propias del Análisis de Redes Sociales para el estudio en profundidad de las relaciones causales de los impactos del turismo identificadas por los participantes. Como se demostrará en las páginas que siguen, este método combinado permite sacar a la luz las causas profundas de los impactos del turismo, señalando nuevas líneas de acción estratégica para la minimización o gestión de los impactos. Se consigue así aunar las ventajas de la participación y las herramientas analíticas propias de las Ciencias Sociales. Ello redunda en una mayor calidad de los resultados finales y, como consecuencia, de los futuros procesos de toma de decisiones en el ámbito de la planificación y la gestión turística.

Este artículo presenta dos objetivos. Por un lado, describe un proceso de participación orientado a la evaluación de los impactos del desarrollo turísticoresidencial, concretado en la identificación de los impactos y sus dinámicas causales. Por otro lado, se ofrece una propuesta para el meta-análisis de los datos producidos durante el proceso participativo. Su novedad reside en la utilización de los programas informáticos de Análisis de Redes Sociales (Ucinet® y NetDraw®) para el tratamiento de mapas causales construidos por los participantes. Los resultados cuantitativos obtenidos de este modo son completados, contextualizados e interpretados a partir de la información obtenida mediante entrevistas en profundidad y revisión documental. A partir de este meta-análisis se produce una nueva información que, devuelta a la comunidad de participantes, puede servirle para conocer e interpretar mejor los cambios sociales que se están produciendo en su territorio.

\section{MÉTODO}

En el marco de rápido cambio estructural experimentado en Pipa, se desarrolló una evaluación participativa de los impactos sociales del desarrollo turístico. El proceso de participación constó de dos fases. En primer lugar, se realizó una serie de entrevistas 
con actores sociales en el ámbito local, regional y nacional. El objetivo de estas entrevistas fue doble. Por un lado, se utilizaron para realizar un Análisis de Partes Interesadas (o Stakeholder Analysis) (DICK, 1997; RAMÍREZ, 1999; CHEVALIER, 2001) con el que identificar a los actores sociales implicados en el sistema turísticoresidencial de Pipa y poder así determinar qué grupos sociales debían estar representados en el proceso de participación. Por otro lado, las entrevistas también se aprovecharon para indagar sobre las diferentes percepciones de los impactos derivados del turismo residencial y para comprender las dinámicas y procesos que los explican. En segundo lugar, se realizó un taller de participación con el objetivo de ofrecer un espacio para la reflexión sobre los impactos sociales del modelo de turismo residencial en Pipa a través de la elaboración de mapas causales.

El Análisis de Partes Interesadas identificó a los principales actores sociales del sistema turístico-residencial mediante un total de 53 entrevistas en profundidad ${ }^{2}$ dirigidas a obtener información en relación a tres dimensiones principales: 1) Dimensión funcional, referida a la identificación de cada uno de los actores que conforman el mapa social del turismo en Pipa, de sus características y funciones; 2) Dimensión estratégica, referida a la posición de poder que ocupa cada uno de los actores, clasificada, a su vez, a partir de cuatro variables fundamentales: a) recursos o capitales de que disponen los actores en su participación en el sistema turísticoresidencial (recursos de tipo financiero, institucional, social, organizacional, simbólico, legal, entre otros); b) influencia o capacidad de utilizar dichos recursos para introducir cambios en el sistema; c) limitaciones u obstáculos en el ejercicio de su influencia; y d) dependencia, tanto hacia otros actores como hacia los beneficios procedentes del turismo residencial; y 3) Dimensión interaccionista, referida a las relaciones que mantienen unos actores con otros y la naturaleza de dicha relación (afinidad, alianza y conflicto). La información recabada a través de las entrevistas en relación a estas cuestiones, permitió identificar los principales sectores sociales cuya representación en el taller de participación se consideraba imprescindible: asociaciones vecinales, hostelería y restauración, promoción urbanística, ámbito académico, ONGs y sector público.

\footnotetext{
${ }^{2}$ El tamaño final de la muestra de entrevistados no fue determinado de forma apriorística sino que, tal y como es habitual en los muestreos no probabilísticos intencionales propios de la investigación cualitativa, fue el resultado de un proceso de saturación teórica (VALLES, 2003).
} 
El taller de participación contó finalmente con la presencia de 22 representantes de estos sectores. Por lo tanto, el modelo de participación seleccionado para este taller no fue de tipo abierto sino controlado, siendo el equipo de investigación quien contactaba con los representantes de los sectores sociales y los convocaba al taller. De este modo, se aseguraba que el taller reflejara adecuadamente la heterogeneidad de intereses e ideologías presentes en la realidad social de Pipa.

Tal y como se ha señalado anteriormente, las entrevistas también sirvieron para recabar las diferentes percepciones acerca de los impactos locales derivados del turismo residencial. Durante las entrevistas, se pidió a cada uno de los entrevistados que identificara y describiera los impactos más significativos. Con esta información el equipo de investigación elaboró un primer listado de impactos que constituyó el punto de partida del taller de participación.

La primera fase del taller consistió en un plenario en el que se desarrolló una tormenta de ideas sobre los impactos derivados del turismo residencial en Pipa, y que fueron añadidos a los impactos identificados a través de las entrevistas. El resultado de la dinámica fue un cuadro con un listado de impactos tanto positivos como negativos (Cuadro 1) consensuado por los participantes.

\begin{tabular}{|c|c|}
\hline $\begin{array}{c}\text { IMPACTOS NEGATIVOS DEL TURISMO } \\
\text { RESIDENCIAL EN PIPA }\end{array}$ & $\begin{array}{c}\text { IMPACTOS POSITIVOS DEL TURISMO } \\
\text { RESIDENCIAL EN PIPA }\end{array}$ \\
\hline $\begin{array}{c}\text { Contaminación visual - discontinuidad } \\
\text { arquitectónica }\end{array}$ & Diversificación de la oferta de alojamiento \\
\hline Deforestación ilegal & Inversiones en Pipa \\
\hline Destrucción de dunas & Recepción de apoyos gubernamentales \\
\hline Destrucción de la mata atlántica & Aumento flujos de turistas \\
\hline Fragmentación paisajística & Mejora del planeamiento urbano \\
\hline Construcción demasiado próximas al mar & Ingresos municipales \\
\hline No respeto de las normas ambientales & Mejora de la calidad de los servicios comerciales \\
\hline Desvalorización de la imagen de Pipa & Aumento de la renta per cápita \\
\hline $\begin{array}{c}\text { Aumento de los precios(de la tierra) - inflación } \\
\text { (inmobiliaria) }\end{array}$ & Capacitación de la mano de obra \\
\hline Inflación (exceso) de oferta de hospedaje & Mejora de las infraestructuras y servicios \\
\hline Turismo de masa - banalización del destino & Generación de empleo \\
\hline Competencia depredadora (o dumping económico) & Conservación de recursos naturales \\
\hline Compra-venta ilegal de terrenos & Intercambio cultural \\
\hline Inestabilidad laboral & Incremento del precio de la tierra \\
\hline $\begin{array}{l}\text { Costes públicos de mantenimiento e } \\
\text { infraestructura sin contraprestaciones }\end{array}$ & $\begin{array}{l}\text { Aumento de la divulgación del destino turístico de } \\
\text { Pipa }\end{array}$ \\
\hline Vida corta del destino turístico & \\
\hline
\end{tabular}


Continuação...

\begin{tabular}{|c|l|}
\hline Construcciones abandonadas - "ciudad fantasma" & \\
\hline Construcciones ilegales & \\
\hline Crecimiento urbano descontrolado & \\
\hline Ciclo corto del destino turístico & \\
\hline Especulación inmobiliaria & \\
\hline Hábitat disperso & \\
\hline $\begin{array}{l}\text { Prioridad de los gastos públicos para accesibilidad } \\
\text { turística en detrimento del saneamiento ambiental }\end{array}$ & \\
\hline
\end{tabular}

FUENTE: ELABORACIÓN PROPIA A PARTIR DE LA INFORMACIÓN PROPORCIONADA POR LOS ACTORES LOCALES.

A partir de la identificación conjunta de impactos, los asistentes procedieron a su discusión y evaluación y, seguidamente, priorizaron ocho que eran los que percibían como más importantes para, en la siguiente fase, elaborar sus mapas causales.

CUADRO 2 - LISTADO DE IMPACTOS PRIORIZADOS IMPACTOS PRIORIZADOS POR LOS PARTICIPANTES

\begin{tabular}{l}
\hline $\begin{array}{l}\text { Prioridad de los gastos públicos para accesibilidad turística en detrimento del saneamiento } \\
\text { ambiental }\end{array}$ \\
\hline Competencia depredadora (o dumping económico) \\
\hline No respeto de las normas ambientales \\
\hline Intercambio sociocultural \\
\hline Crecimiento urbano desordenado \\
\hline Aumento de la divulgación del destino turístico de Pipa \\
\hline Ciclo corto del destino turístico \\
\hline Deforestación ilegal \\
\hline FUENTE: ELABORACIÓN PROPIA A PARTIR DE LA INFORMACIÓN PROPORCIONADA POR \\
LOS ACTORES LOCALES.
\end{tabular}

Utilizando estos ocho impactos priorizados (a partir de ahora impactos $_{p}$ ) como elementos de partida, se elaboró un mapa causal (Gráfico 1) para cada uno de ellos. Un mapa causal es una subclase de mapa cognitivo cuyo fin es mostrar el conocimiento en un ámbito específico a través de enlaces de causalidad (WEICK, 1979; SCAVARDA et al., 2004). Las opiniones, ideas y/o temas clave del problema que se está investigando son representados como nodos que están conectados por flechas que muestran la dirección de la causalidad. Como muestra el Gráfico 1, los nodos y sus relaciones causales dan al mapa causal forma de reloj de arena. En el centro del mismo se sitúa el impacto priorizado en el que confluyen, a través de líneas verticales, todas las relaciones causales situadas por debajo y del que surgen todos los efectos dispuestos por arriba. De esta forma se ordena la secuencia de causalidad de un impacto determinado. Por 
ejemplo, la "Ignorancia de la leyes" es causa de la "Falta de fiscalización" que a su vez es causa del "Crecimiento urbano descontrolado".

GRÁFICO 1 - EJEMPLO DE MAPA CAUSAL

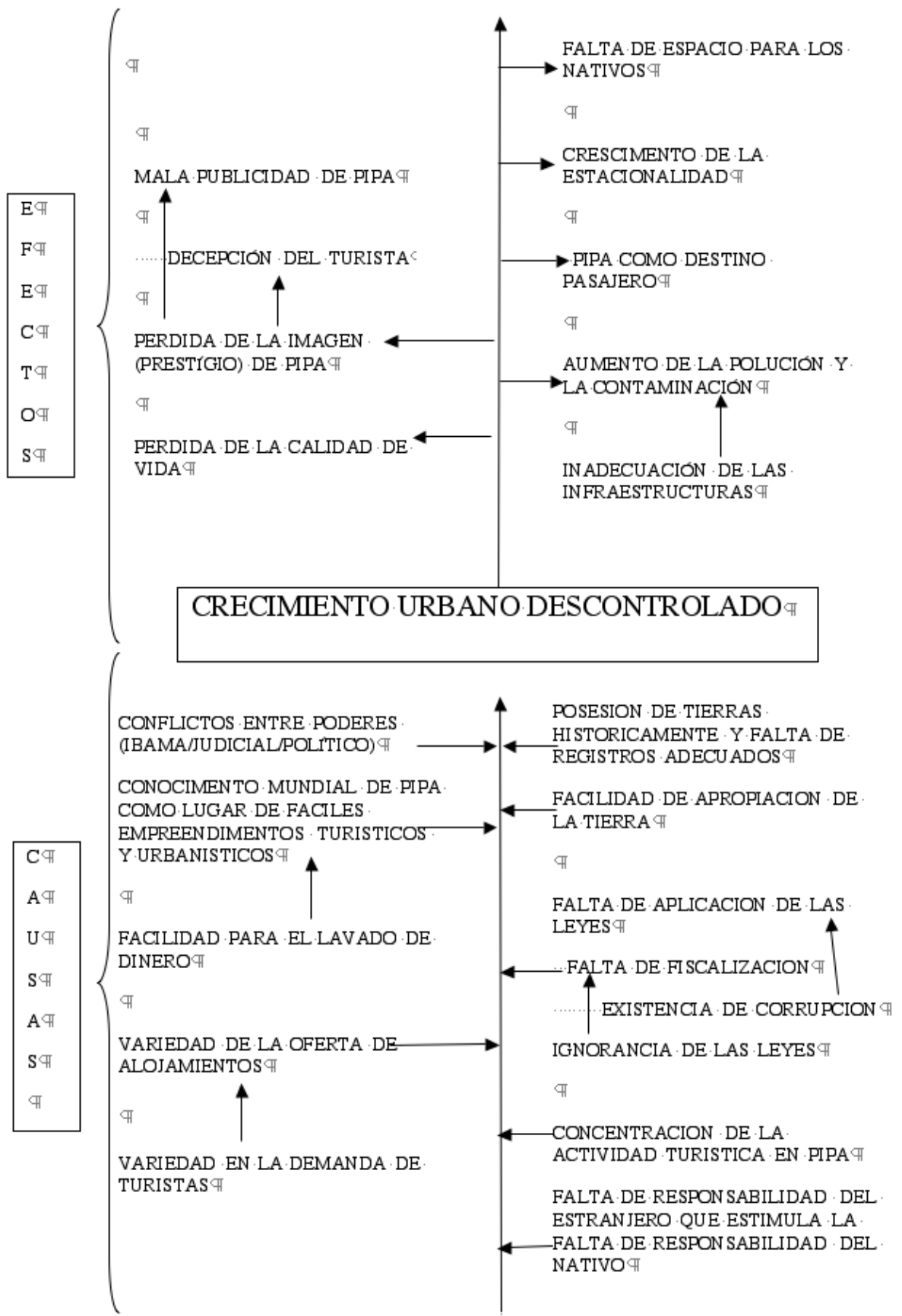

FUENTE: ELABORACIÓN PROPIA A PARTIR DE LA INFORMACIÓN PROPORCIONADA POR LOS ACTORES LOCALES.

Resulta habitual crear los mapas causales mediante reuniones de grupo (ACKERMAN y EDEN, 2001; VO, SCOTT y COURTNEY, 2005). Creado de forma 
colectiva, el mapa causal representa un punto de vista común sobre el tema analizado una vez se han discutido las diferentes posiciones. Con este método es mucho más fácil que con otras técnicas cualitativas conseguir acuerdos y llegar a un consenso entre los participantes (ALBINO, KÜHTZ y SCOZZI, 2002). Además, el contenido del mapa causal colectivo utiliza la terminología de los participantes en lugar de la que impone el propio investigador (WEICK y BOUGON, 1986). Igualmente, trabajar con datos procedentes de múltiples individuos ayuda a reducir la presencia de sesgos.

Tal y como se ha indicado anteriormente, en torno al desarrollo local del turismo residencial aparece una amplia variedad de actores sociales. Esta heterogeneidad de grupos e intereses fundamenta la selección de una estrategia participativa en la construcción de los mapas causales. Además, el esfuerzo deliberativo y la heterogeneidad de los participantes favorecen que los mapas causales elaborados no se limiten a recoger las causas y los efectos más evidentes y generales que se derivan de los impactos. . Por el contrario, la heterogénea composición del grupo y la deliberación hacen emerger los elementos causales de mayor capacidad explicativa (JAMAL y GETZ, 1995; BROHMAN, 1996; MAZÓN et al., 1996; TOSUN y TIMOTHY, 2003, SCAVARDA et al., 2004; EVANS, 2005).

Con respecto a la utilización de la metodología de mapas causales en el ámbito del turismo, cabe destacar el trabajo de Nash (2006) y Woodside (2007). Nash (2006) utiliza esta metodología para identificar las cuestiones clave de interés para los diferentes actores públicos y privados involucrados en el desarrollo del turismo rural en tres destinos de Escocia. Woodside (2007) utiliza mapas de causalidad en el estudio de la psicología y el comportamiento del turista. El autor destaca la importancia de considerar las influencias indirectas para comprender mejor los procesos de pensamiento que condicionan la conducta de ocio de viajes ilustrados en el mapa causal analizado. Lo señalado por Woodside retoma una idea expuesta en la década de los ochenta por Mathieson y Wall (1982) en la que apuntaban la dificultad de trazar el complejo orden de flujos de repercusión derivado de los impactos locales del desarrollo turístico. En ambos casos, se hace mención a la intrincada y compleja red de relaciones directas e indirectas que es necesario analizar para entender las relaciones causales. 


\section{META-ANÁliSiS DE LOS RESULTADOS DEL PROCESO DE PARTICIPACIÓN}

El análisis aislado de cada uno de los mapas causales elaborados mejora el proceso de evaluación al desarrollar y organizar la cadena causal de los impactos permitiendo centrar la atención en las causas de fondo de los problemas (EVANS, 2005). No obstante, los mapas causales también pueden ser analizados y comparados elaborando un mapa causal conjunto o una red. El uso de programas informáticos proporciona una mayor capacidad para esta tarea. El Análisis de Redes Sociales (ARS) ha sido tradicionalmente aplicado en el estudio de las interacciones entre los miembros de una comunidad u organización (SCOTT y CARRINGTON, 2011; SCOTT, BAGGIO y COOPER, 2008). Sin embargo, es una metodología adecuada para el análisis de los mapas causales, ya que, según lo indicado por Nelson y Mathews (1991, p. 380) para el campo de diagnóstico de las organizaciones, "utiliza la mecánica matricial similar" ${ }^{3}$. En esta línea, existen trabajos en el campo de la toma de decisiones grupales. Por ejemplo, Ward y Reingen (1990) aplican esta combinación para estudiar cómo la estructura social influye en el conocimiento compartido y cómo ésta influye en la elección del consumidor. Un poco más allá, Weber y Manning (2001) aplican el programa informático Ucinet ${ }^{\circledR}$ al análisis de mapas causales con el fin de descubrir patrones de consistencia interna y similitud conceptual (clusters) en el contexto del liderazgo escolar. Sin embargo, en ninguno de las investigaciones que utilizan mapas causales se ha tenido en cuenta el análisis sistemático de las influencias indirectas entre los nodos de la red tal y como destaca Woodside (2007). No tener en cuenta las relaciones indirectas conlleva ignorar la naturaleza compleja e intrincada de los procesos causales que tienen lugar alrededor de los impactos sociales del turismo.

Para trazar y hacer analizables los complejos procesos causales de los impactos locales del turismo residencial, esta investigación presenta como novedad la incorporación al proceso de diagnóstico de un meta-análisis de los mapas causales a partir de herramientas propias del Análisis de Redes Sociales, mediante el uso de los programas informáticos Ucinet® y NetDraw ${ }^{\circledR}$. Estos programas permiten combinar los diferentes mapas causales y producir una red de procesos causales inducidos por el

\footnotetext{
${ }^{3}$ Para más información sobre el software de soporte para el análisis de los mapas de causalidad, consultar Narayanan y Armstrong (2005).
} 
turismo residencial en Pipa (Gráfico 2). Esta red es posible gracias a la existencia de elementos comunes en los distintos mapas causales. Por ejemplo, el nodo "Pérdida de la calidad de vida" (cuya etiqueta en el gráfico es 109) está presente en tres mapas causales: Crecimiento urbano desordenado, No respeto de las normas ambientales y Competencia depredadora. Así, tal y como se muestra en el Gráfico 2, compartir este nodo permite ligar los tres mapas causales contribuyendo a construir una red causal más amplia e interconectada.

\section{GRÁFICO 2 - RED CAUSAL DE LOS IMPACTOS DEL TURISMO RESIDENCIAL EN PIPA}

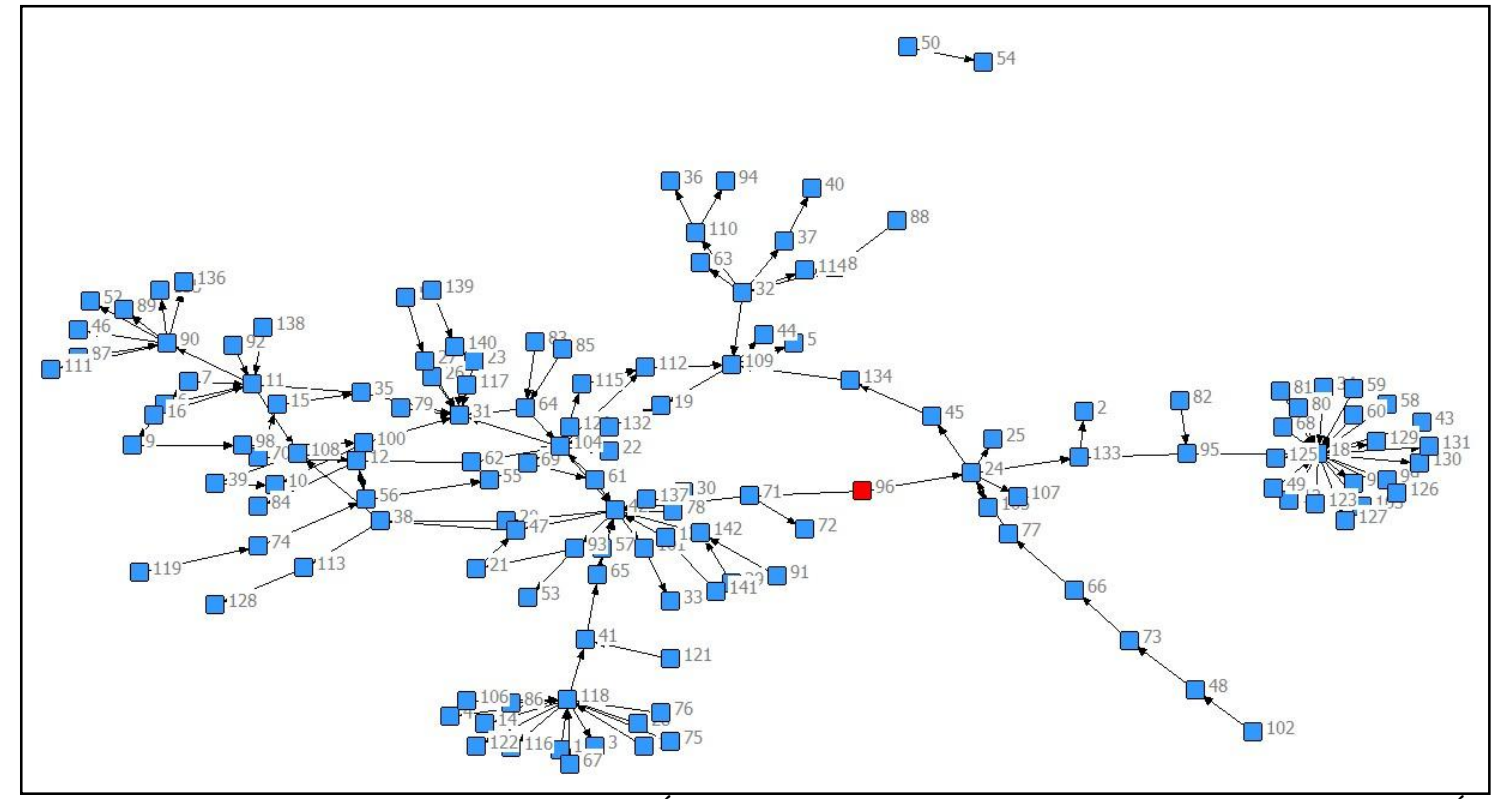

FUENTE: NETDRAW Y YLABORACIÓN PROPIA A PARTIR DE LA INFORMACIÓN PROPORCIONADA POR LOS ACTORES LOCALES.

NOTA: EL NODO 96 (MARKETING Y PUBLICIDAD DE LAS GRANDES EMPRESAS) DESTACA EN ROJO POR SER EL NODO CON MAYOR INFLUENCIA DE LA RED.

Una vez obtenida la red, es posible proceder a su análisis. El estudio de la red causal no debe limitarse al estudio de las influencias directas que cada nodo ejerce en los elementos de su entorno inmediato. Por el contrario, si se pretende entender el complejo entramado de relaciones causales, es necesario atender a las influencias indirectas. Éstas permiten comprender cómo cada nodo influye, a través de otros, en el resto del sistema. De este modo, es posible identificar los nodos más influyentes del conjunto de la red (GARCÍA-ANDREU, 2008).

Para analizar la red causal teniendo en cuenta las influencias indirectas se utiliza el indicador estadístico de cercanía ejercida o outcloseness mediante el programa informático Ucinet巴. El indicador de cercanía mide la capacidad de cada nodo de 
alcanzar al resto de nodos de la red utilizando para ello nodos intermedios (relaciones indirectas). Así, son más centrales los nodos con un mayor índice de cercanía o, en otras palabras, aquellos nodos que, por su posición en la red, influyen sobre el mayor número de nodos. Este tipo de análisis permite la identificación de la base causal de los impactos $_{p}$.

En el marco de un proceso de planeamiento o intervención social, esta información es de especial relevancia porque, al actuar sobre estos nodos basales, se ocasionaría un mayor efecto sobre el conjunto del sistema.

Sirva de ejemplo el resultado obtenido en la investigación realizada en Pipa, donde los datos arrojados por el análisis de la red causal identificaron las causas centrales (outcloseness $>1,000^{4}$ ) de los impactos del desarrollo turístico-residencial (Cuadro 3).

CUADRO 3 - CAUSAS CENTRALES DE LOS IMPACTOS DEL TURISMO RESIDENCIAL EN PIPA (OUTCLOSENESS >1,000)

\begin{tabular}{|c|c|c|c|}
\hline $\begin{array}{l}\text { Etiqueta } \\
\text { en la red }\end{array}$ & Ranking & Nodo & Valor outcloseness \\
\hline 96 & 1 & Marketing y publicidad de las grandes empresas & 1,109 \\
\hline 1 & 2 & $\begin{array}{c}\text { La estructura pública no acompaña la } \\
\text { estacionalidad del volumen de basura generado en } \\
\text { la región }\end{array}$ & 1,099 \\
\hline 17 & 3 & Carencia de servicios públicos en áreas urbanas & 1,099 \\
\hline 106 & 4 & $\begin{array}{l}\text { El municipio no tiene preparada una estructura para } \\
\text { la implementación del alcantarillado y de una malla } \\
\text { viaria }\end{array}$ & 1,099 \\
\hline 76 & 5 & Falta de recursos y medios del poder público & 1,099 \\
\hline 75 & 6 & Falta de recursos de comunicación & 1,099 \\
\hline 28 & 7 & Connivencia del poder público & 1,099 \\
\hline 67 & 8 & Falta de información & 1,099 \\
\hline 118 & 9 & $\begin{array}{l}\text { Prioridad de los gastos públicos para accesibilidad } \\
\text { turística en detrimento del saneamiento ambiental }\end{array}$ & 1,092 \\
\hline 121 & 10 & $\begin{array}{c}\text { Problemas culturales } \\
\end{array}$ & 1,025 \\
\hline 41 & 11 & Desinterés y falta de compromiso cívico-social & 1,018 \\
\hline 69 & 12 & Falta de inversión en educación & 1,013 \\
\hline 119 & 13 & Priorización de los intereses políticos y personales & 1,012 \\
\hline 71 & 14 & $\begin{array}{l}\text { Falta de planeamiento } \\
\end{array}$ & 1,011 \\
\hline 65 & 15 & Impunidad & 1,011 \\
\hline 62 & 16 & $\begin{array}{l}\text { Falta de colaboración entre la comunidad, el poder } \\
\text { público y los empresarios }\end{array}$ & 1,01 \\
\hline 83 & 17 & Fiscales mal capacitados & 1,01 \\
\hline 85 & 18 & Ignorancia de las leyes & 1,01 \\
\hline 29 & 19 & Construcción de condominios & 1,008 \\
\hline
\end{tabular}

\footnotetext{
${ }^{4}$ Cabe señalar que el rango de valores de outcloseness de esta red oscila entre los valores 0,704 y 1,109.
} 
Continuação...

\begin{tabular}{|c|c|c|c|}
\hline 91 & 20 & Intereses económicos particulares & 1,008 \\
\hline 141 & 21 & Venta de madera & 1,008 \\
\hline 16 & 22 & Bajas restricciones ambientales & 1,006 \\
\hline 138 & 23 & Valorización escénica del paisaje & 1,006 \\
\hline 92 & 24 & Inversiones en publicidad & 1,006 \\
\hline 74 & 25 & Falta de priorización de inversiones públicas & 1,005 \\
\hline 61 & 26 & Falta de educación e conciencia ambiental & 1,005 \\
\hline 132 & 27 & $\begin{array}{l}\text { Relación de cuanto menor es la formación de la } \\
\text { población mayor será su manipulación }\end{array}$ & 1,003 \\
\hline 64 & 28 & Falta de fiscalización y monitorización & 1,003 \\
\hline 120 & 29 & Priorización de inversiones en otros sectores & 1,003 \\
\hline 124 & 30 & Quemas & 1,001 \\
\hline 78 & 31 & Falta de respeto al plan director & 1,001 \\
\hline 137 & 32 & Utilización de las dunas para paseos en buggy & 1,001 \\
\hline 142 & 33 & Venta de la tierra & 1,001 \\
\hline 57 & 34 & $\begin{array}{l}\text { Falta conocimiento de la población, así como el } \\
\text { poder de los grandes grupos que influyen en el plan } \\
\text { director }\end{array}$ & 1,001 \\
\hline
\end{tabular}

Una vez identificados estos nodos centrales, es necesario contextualizar esta información en las dinámicas locales y globales del turismo residencial para dotar de sentido al análisis cuantitativo. Para ello, se integra en este meta-análisis el conocimiento adquirido por el equipo de investigación a través de las entrevistas realizadas durante el trabajo de campo y la revisión documental previa. A partir de aquí, es posible establecer, de nuevo con la cooperación de los agentes locales, futuras líneas de actuación estratégica.

No es posible en este artículo interpretar la totalidad de causas identificadas por este análisis, por lo que se ha focalizado la atención en aquellas que obtienen puntuaciones más altas en el ranking. De este modo, se han considerado como tales los primeros nueve nodos mostrados en el Cuadro 3 ya que sus valores están muy próximos y se distancian significativamente el valor del siguiente nodo del ranking (nodo 10).

\section{CONTEXTUALIZACIÓN E INTERPRETACIÓN DE LAS CAUSAS CENTRALES DE LOS IMPACTOS DEL TURISMO RESIDENCIAL EN PIPA}

Los nodos que tras el análisis de cercanía aparecieron como más influyentes pueden ser agrupados en las siguientes dimensiones: a) la importancia del efecto del 
marketing y la publicidad de las grandes empresas de turismo residencial (1), b) el déficit democrático en la gestión local y debilidad institucional (5, 6, 7 y 8), y c) déficit de infraestructuras y su desigual distribución socioespacial (2, 3, 4 y 9).

La causa que ejerce mayor influencia dentro del sistema causal construido por los participantes del taller es Marketing y publicidad de las grandes empresas (en color rojo en el gráfico 2). Esta causa hace referencia al modo en que los promotores turístico-residenciales, a través de su capacidad mediática, venden la ilusión de que controlan una ingente masa de compradores europeos en busca de una segunda residencia en el nordeste brasileño. Se presentan ante la comunidad local y sus representantes políticos como atractores de una demanda internacional de segundas residencias que va a traer consigo innumerables riquezas para el conjunto de la población $\mathrm{y}$, de forma más individual, sobre determinados actores situados en posiciones clave en los proceso de toma de decisiones urbanísticas locales o estatales. Estos promotores internacionales construyen un discurso en el que se posicionan como los conectores entre la demanda potencial y el futuro desarrollo turístico del municipio. Esta realidad construida les confiere un poder y una capacidad de negociación enorme tanto sobre los propietarios individuales de terrenos como ante los representantes políticos locales.

En realidad, ese control de la demanda internacional es ficticio, como así lo ha demostrado la caída radical en la venta de viviendas en este destino a partir de 2008. En el marco de una competencia turística mundial entre destinos, las demandas turísticas internacionales se caracterizan por su extrema volatilidad. En otras palabras, esta demanda internacional no está, se construye en una disputa dentro del sector de la promoción urbanística internacional. Por tanto, no se puede poseer o controlar por parte de estos promotores turístico-residenciales. Es una ficción, propia de la actual fase del capitalismo (VERDÚ, 2003). Al mismo tiempo que los promotores turísticoresidenciales exhiben su supuesto control de una demanda internacional ilimitada también se presentan ante los mercados emisores como poseedores de una oferta turístico-residencial perfectamente construida y preparada para recibirlos. En la publicidad, ofrecen unos paraísos turístico-residenciales que satisfacen las demandas postmodernas de exotismo, seguridad física y alta rentabilidad inversora. También estos paraísos han demostrado ser una ficción. En gran parte, ni siquiera estaban construidas 
las infraestructuras mínimas y necesarias para iniciar los proyectos que ya se vendían como hechos. Aparece así un turismo de ficción. Una ficción construida a través de discursos orales, escritos y audiovisuales $\mathrm{y}$, que aprovechándose de Internet, se convierte en un capital simbólico transportable y accesible a casi cualquier lugar del mundo. Este discurso del turismo de ficción otorga un poder enorme a estos promotores urbanísticos, tanto sobre la demanda como sobre la oferta.

Este poder de los promotores foráneos es una herramienta de presión y negociación ante el poder político local que controla una parte importante de las decisiones referentes al ámbito del planeamiento urbanístico. El discurso del turismo residencial presenta al sector internacional como un motor de desarrollo socioeconómico para la comunidad y como el nexo que liga estas periféricas helioterrae con los mercados globales. Al mencionado supuesto o ficticio beneficio comunitario se suman los beneficios particulares que determinados actores políticos y económicos locales pueden conseguir a cambio de tierras turísticamente interesantes o por su capacidad de influencia política en las decisiones urbanísticas a tomar por la prefeitura. La preeminencia de intereses particulares sobre los colectivos constituye el déficit democrático en la gestión local identificado por los participantes y que sostiene el entramado de decisiones que favorecen a las empresas promotoras y a las elites locales. Además, los intereses particulares también se han visto fortalecidos frente al interés general a consecuencia de la connivencia del poder público en la escasa fiscalización de los proyectos turístico-residenciales y en la impunidad ambiental de las acciones de sus promotores. La falta de información en la gestión local sería una manifestación más de una gestión pública espuria. A estas circunstancias se añade la debilidad financiera municipal y el déficit de cuadros administrativos formados, especialmente en municipios pequeños y periféricos, como es el caso de Tibau do Sul.

En lo referente a la carencia de servicios públicos, que se ve aumentada por la estacionalidad turística, cabe señalar el hecho de que en la nueva configuración urbanística de Pipa se observa cómo las nuevas infraestructuras y servicios se están localizando de acuerdo con los intereses y necesidades de los proyectos turísticoresidenciales, configurando una nueva lógica territorial. Mientras las zonas habitadas por los habitantes oriundos de Pipa siguen padeciendo graves deficiencias, las nuevas carreteras o el alcantarillado se construyen en torno a estos nuevos espacios urbanos que 
además se sitúan fuera del casco tradicional, dado que el suelo allí es más barato. Con el paso del tiempo, surge la paradoja de que, al estar vacíos estos condominios por falta de demanda, estas nuevas infraestructuras están absolutamente infrautilizadas. Esos nuevos espacios centran el interés económico y político, concentran la inversión y se presentan globalmente como la nueva Pipa dotada de nuevos significados culturales ajenos a los culturalmente propios de la Pipa anterior.

\section{CONSIDERACIONES FINALES}

Hasta este momento esta propuesta metodológica ha conseguido, en primer lugar, identificar a través de un proceso participativo los elementos causales con mayor capacidad explicativa de los impactos derivados del turismo residencial en el caso de estudio. En segundo lugar, el meta-análisis realizado sobre estos datos ha permitido medir la intensidad con que determinadas variables afectan al sistema turísticoresidencial, así como interpretar esta información cuantitativa a partir de los conocimientos generados durante el trabajo de campo.

La aplicación de los programas informáticos propios del Análisis de Redes Sociales al análisis de mapas causales realizados en el marco de procesos participativos ha permitido realizar una evaluación de la compleja estructura causa-efecto que se esconde detrás de los impactos locales del turismo. Uno de los resultados de este análisis es la capacidad de identificar de forma sistemática los nodos con mayor potencia explicativa de la red causal de impactos. Mediante este análisis, que tiene como fuente el conocimiento local, se produce un conocimiento nuevo, inexistente con anterioridad a la investigación, que emerge de la combinación de conocimientos heterogéneos organizados y plasmados en forma de mapas causales. Este conocimiento nuevo puede servir a los actores sociales para conocer e interpretar mejor los procesos y fenómenos sociales que tienen lugar en su comunidad. Así, la finalidad última de los resultados del meta-análisis es demarcar áreas de actuación que, en un posterior taller participativo, sean discutidas y tomadas como punto de partida de propuestas de acción estratégica. 
El análisis de la red causal del turismo residencial en el caso de Pipa ha permitido descubrir cómo la lógica global se impone sobre la lógica local. Aquí se ha usado el término lógica para hacer referencia a una forma de producir el espacio con un orden que responde a unos determinados intereses. Cuando esos intereses son mayoritariamente foráneos, ligados a fenómenos globales como el turismo y la circulación internacional de capitales, se puede interpretar esa forma de ver y ordenar el territorio como una lógica global. La lógica local respondería a intereses y grupos locales aún cuando se entiende que esta no es homogénea sino plural y polifónica.

La imposición de la lógica global sobre la local se demuestra mediante la desigual distribución de infraestructuras y servicios que benefician a los nuevos territorios turísticos en detrimento de los espacios excluidos de la expansión turística. En otras palabras, el modelo de desarrollo turístico implementado en Pipa se orienta a favorecer la acumulación de capital o beneficios por parte del capital privado internacional (lógica global) sobre la distribución social de los beneficios (lógica local). La imposición de la lógica global se articula, por un lado, con el apoyo de las elites económicas y políticas locales y, por otro, se fundamente en la causa basal identificada por el análisis, esto es, el marketing y publicidad de las grandes empresas. No obstante, sobre esta causa basal se construye un discurso fundamentado en una ficción: la capacidad de las grandes promotoras de controlar una demanda turístico-residencial. A partir de la deconstrucción de este discurso - del reconocimiento de lo ilusorio del control de las empresas sobre la demanda -, la población local podría articular un nuevo discurso que primase la lógica local sobre la lógica global, dando lugar a nuevas propuestas para reorientar el modelo de desarrollo turístico hacia una priorización de la distribución social de los beneficios sobre los procesos de acumulación.

\section{REFERENCIAS}

ACKERMAN, F; EDEN, C. Contrasting single user and Networked Group decisión support systems for strategy making. Group Decisión and Negotiation, v. 10, p. 47-66, 2001.

ALBINO, V.; KÜHTZ, S.; SCOZZI, B. Cognitive maps and the sustainable development in the Mediterranean region, Proceedings of local resources and global trades. In: INCOSUSW MEETING, Rabat (Morocco), 2002. Disponible en: <http://ressources.ciheam.org/om/pdf/a57/04001986.pdf>. Acceso el: 18/3/2012. 
ALEDO, A. De la tierra al suelo: la transformación del paisaje y el nuevo turismo residencial. Arbor, v. 184, n. 729, p. 99-113, 2008.

AP, J. Residents Perceptions on Tourism Impacts. Annals of Tourism Research, v. 19, n. 4, p. 665-690, 1992.

BANCO CENTRAL DO BRASIL. Evolução do IDH das Grandes Regiões e Unidades da Federação. Boletim Regional do Banco Central do Brasil, Enero, 2009. Disponible en: <http://www.bcb.gov.br/pec/boletimregional/port/2009/01/br200901b1p.pdf>. Acceso en: 19/12/2012.

BANCO DO NORDESTE. PRODETUR/NE I: Resultados. 2006. Disponible en: $<$

>. Acceso el: 16/7/2012

BANCO DO NORDESTE. PRODETUR/NE II. 2009. Disponible en:

<http://www.bnb.gov.br/content/aplicacao/PRODETUR/Prodetur_ne2/gerados

/situacao_atual.asp>.Acceso el: 16/7/2012.

BANCO MUNDIAL. Public Involvement in Environmental Assessment: requirements, opportunities and issues. Environmental Assessment Sourcebook Update. Washington DC: World Bank, 1993.

BECKER, D. R.; HARRIS, C. C.; MCLAUGHLIN, W. J.; NIELSEN, E. A. A participatory approach to social impact assessment: the interactive community forum. Environmental Impact Assessment Review, v. 23, p. 367-382, 2003.

BECKER, H. A.; VANCLAY, F. The International Handbook of Social Impact Assessment: conceptual and methodological advances. Cheltenham: Edward Elgar Publishing Limited, 2003.

BLÁZQUEZ, M.; CAÑADA, E. Turismo placebo. Nueva colonización turística: del Mediterráneo a Mesoamérica y El Caribe. Lógicas espaciales del capital turístico. Managua: EDISA, 2011.

BLÁZQUEZ, M.; CAÑADA, E.; MURRAY, I. (2011). Búnker playa-sol. Conflictos derivados de la construcción de enclaves de capital transnacional turístico español en el Caribe y Centroamérica. Scripta Nova: revista electrónica de geografía y ciencias sociales, v. XV, n. 368, 2011. Disponible en: < >. Acceso en: 31/1/2012.

BRAMWELL, B. Coastal mass tourism: Diversification and sustainable development in southern Europe. Clevedon: Channel View Pub, 2004.

BROHMAN, J. New directions in tourism for third world development. Annals of Tourism Research, v. 23, n.1, p. 48-70, 1996. 
BURDGE, R. A community guide to social impact assessment. Middelton (WI): Social Ecology Press, 1995.

BURDGE, R.; VANCLAY, F. Social impact assessment: A contribution to the state-ofthe-art series. Impact Assessment, v. 14, n. 1, p. 59-86, 1996.

CHEVALIER, J. Stakeholder analysis and natural resource management. Ottawa: Carelton University. 2001. Disponible en: <http://www1.worldbank.org/publicsector/politicaleconomy/November3Seminar/Stakeh lder\%20Readings/SA-Chevalier.pdf >. Acceso en: 24/11/2012.

CRANDALL, L. The social impact of tourism on developing regions and its measurement. In: RITCHIE, J. R. B.; GOELDNER, C. R. (Org.) Travel, tourism, and hospitality research. A handbook for managers and researchers. New York: John Wiley and Sons, 1987. p. 373-383.

CRUZ, R. C. Política de turismo e território. São Paulo: Contexto, 2000.

DANTAS, E. W. C. Imaginário social nordestino e políticas de desenvolvimento do turismo no nordeste brasileiro. GEOUSP - Espaço e Tempo, n. 22, p. 9 - 30, 2007.

DANTAS, E. W. C.; FERREIRA, A; LIVRAMENTO, M. C. Turismo e imobiliário nas metrópoles. Rio de Janeiro: Letra Capital, 2010.

DEMAJOROVIC, J.; ALEDO, A.; LANDI, B.; KONDO, A. L. M. Complejos Turísticos Residenciales. Análisis del crecimiento turístico residencial en el Mediterráneo español y en el Litoral Nordestino (Brasil) y sus impactos socioambientales. Estudios y perspectivas en Turismo, v. 20, p. 772-796, 2011.

DICK, B. Stakeholder Analysis. Resource papers in Action Research, 1997. Disponible en: <http://www.scu.edu.au/schools/gcm/ar/arp/stake.html>. Acceso en: 26/3/2012.

EVANS, J. R. Total quality management, organization, and strategy (4. ed.). Mason, OH: Thomson South-Western, 2005.

FONSECA, M A. P.; ADEMIR, A. A racionalidade da urbanização turística em áreas deprimidas: o espaço produzido para o visitante. Scripta Nova: revista electrónica de geografía y ciencias sociales, Barcelona, v. 9, n. 194, 2005. Disponible en: $<$ >. Acceso en: 2/7/2012.

GARCÍA-ANDREU, H. Sociedad, turismo y medio ambiente: Una propuesta desde la Sociología para el diagnóstico y la reorientación de los municipios turísticoresidenciales del litoral español. 448 p. Tesis (Doctorado en Sociología) - Departamento de Sociología I, Universidad de Alicante, Alicante, España, 2008. 
GARCÍA-ANDREU, H.; ALEDO, A.; ORTIZ, G. Análisis de mapas causales de impactos del turismo residencial. Empiria: revista de metodología de Ciencias Sociales, Madrid, v. 20, p. 61-86, 2010.

GREENPEACE. Los nuevos conquistadores. Multinacionales españolas en América Latina. Impactos económicos, sociales y medioambientales. Madrid: Greenpeace España, 2009.

HIERNAUX, N. D. Las segundas residencias en México. Un balance. México: PyV, 2010.

INSTITUTO BRASILEIRO DE GEOGRAFÍA E ESTATÍSTICA. Disponible en: <http://www.ibge.gov.br/cidadesat/link.php?codmun=241420>. Acceso en: 24/01/2013.

JAMAL, T. B.; GETZ, D. Collaboration theory and community tourism planning. Annals of Tourism Research, v. 22, n.1, p. 186-204, 1995.

LEÓN, Y. M. The impact of tourism on rural livelihoods in the Dominican Republic's coastal areas. Journal of Development Studies, v. 43, n. 2, p. 340-359, 2007.

LOLOUM, T. Con-dominium. Turismo residencial internacional y desarrollo inmobiliario en el nordeste brasileño. Tesis (Máster, Planificación Integrada para el Desarrollo Local y la Gestión Ambiental) - Universidad de Lleida/CIHEAM, Zaragoza, España, 2010.

MATTEUCCI, X.; LUND-DURLACHER, D.; BEYER, M. The socio-economic and environmental impacts of second home tourism. In: KELLER, P.; BIEGER, T. (Orgs.), Real estate and destination development in tourism: Successful strategies and instruments. Berlin: Erich Schmidt, 2008. p. 146-160.

MATHIESON, A.; WALL, G. Tourism economic, physical and social impacts. Longman: Harlow, 1982.

MAZÓN, T. Inquiring into residential tourism: The Costa Blanca case. Tourism and Hospitality Planning \& Development, v. 3, n. 2, p. 89-97, 2006.

MAZÓN, T.; ALEDO, A.; ARROCHA, A.; MARTÍNEZ, E. El turismo inmobiliario en la provincia de Alicante: análisis y propuestas. Alicante: Diputación de Alicante, 1996.

MONTI, E. La crisis económica internacional de 2008 y el turismo: Efectos y medidas de respuesta en Rio Grande do Norte, Brasil. Investigaciones Turísticas, v. 1, n. 1, p. 93-106, 2011.

MUNRES. Programa MUNRES: Programa de Revitalización de Municipios con Turismo Residencial. Investigaciones básicas. Alicante: Instituto Universitario de Geografía y Diputación de Alicante, 1995. 
MURPHY, P. E. Tourism: a Community Approach. Londres: Routledge, 1985.

NAREDO, J. M. El Modelo Inmobiliario Español y sus consecuencias. Revista Habitat. Ciudades para un Futuro más Sostenible Boletín $\mathbf{C F}+\mathbf{S}$ 44, 2010. Disponible en: <http://habitat.aq.upm.es/boletin/n44/ajnar.html>. Acceso en: 16/2/2012.

NARAYANAN, V. K.; ARMSTRONG, D. J. Causal Mapping for Research in Information Technology. Hershey, PA: IGI Global, 2005.

NASH, R. Casual Network Methodology. Tourism Research Applications. Annals of Tourism Research, v. 33, n. 4, p. 918-938, 2006.

NAVALÓN, R. Planeamiento urbano y turismo residencial en los municipios litorales de Alicante. Alicante: Instituto de Cultura Juan Gil-Albert, 1995.

NELSON, R. E.; MATHEWS, K. M. Cause maps and Social Network Analysis in Organizational Diagnosis. Journal of Applied Behavioral Science, v. 27, n. 3. p. 379397, 1991.

O'REILLY, K. The Rural Idyll, Residential Tourism, and the Spirit of Lifestyle Migration. In: ASA CONFERENCE, 10-13 Abril, Londres, London Metropolitan University, 2007.

O'REILLY, K. (2009). Hosts and guests, guests and hosts: British residential tourism in the Costa del Sol. In: OBRADOR, P.; CRANG, M.; TRAVLOU, P. (Orgs.). Cultures of Mass Tourism: Doing the Mediterranean in the Age of Banal Mobilities. Farnham: Ashgate, 2009. p. 129-142.

ORTIZ, G.; GARCÍA, H.; JUAN, P.; ALEDO, A. Epistemological and ethical dilemmas of public participation on residential tourism planning. PASOS: Revista De Turismo y Patrimonio Cultural, v. 5, n. 3, p. 323-329, 2007.

ORTIZ, G. Participación ciudadana, investigación social y desarrollo local: una propuesta metodológica aplicada a un municipio turístico-residencial. 594 p. Tesis (Doctorado en Sociología) - Departamento de Sociología I, Universidad de Alicante, Alicante, España, 2009.

PEREIRA, R. C. Un análisis para identificar los espacios turísticos y no turísticos en la relación entre turismo y comunicación en Brasil. Estudios y Perspectivas en Turismo, v. 21, p. 126-140, 2012.

RAMÍREZ, R. Stakeholder analysis and conflict management. In: D. BUCKLES (Org.). Cultivating peace: Conflict and Collaboration in Natural Resource Management. Washington: World Bank Publications, 1999. p. 101-126. 
RIBEIRO L. C. Estudo comparativo sobre o papel das atividades imobiliárias na transformação do espaço social das metrópoles nordestinas: Salvador, Recife, Natal e Fortaleza. Informe de investigación. Rio Grande do Norte: Observatório das Metrópoles, 2009. Disponible en:

$<$

Acceso en: 4/6/2012.

ROBERTS, R. Involving the public. In: BECKER, H. A,; VANCLAY, F. The International Handbook of Social Impact Assessment. Cheltenham: Edward Elgar Publishing Limited, 2003. p. 258-277.

SCAVARDA, A. J.; BOUZDINE-CHAMEEVA, T.; MEYER, S.; HAYS, J. M.; HILL, A. V. A Review of the Causal Mapping Practice and Research Literature. In: SECOND WORLD CONFERENCE ON POM AND 15 ANNUAL POM CONFERENCE, Cancun, Mexico, 2004.

SCOTT, N.; BAGgiO, R.; COOPER, C. Network Analysis And Tourism. From Theory To Practice. Clevedon, Channel View, 2008.

SCOTT, J.; CARRINGTON, P. J. The Sage Handbook of Social Network Analysis. Thousand Oaks: Sage publications, 2011.

SILVA, K. O.; FONSECA, M. A. P. A produção de residência secundária no litoral oriental Potiguar: Turismo e capital imobiliário. Caderno Virtual de Turismo, v. 10, n. 1, p. 50-63, 2010.

TAYLOR, C; BRYAN, C.; GOODRICH, C. Social Assessment: Theory, Process and Techniques. Middleton (WI): Social Ecology Press, 2004.

TOSUN, C.; TIMOTHY, D. J. Arguments for community participation in the tourism development process. The Journal of Tourism Studies, v. 14, n. 2, p. 2-15, 2003.

VALLES, M. S. Técnicas cualitativas de investigación social: reflexión metodológica y práctica profesional. Madrid: Síntesis Sociología, 2003.

VANCLAY, F. International Principles for Social Impact Assessment. Impact Assessment and Project Appraisal, v. 21, n. 1, p. 5-11, 2003.

VERA, J. F. Análisis Territorial del Turismo: una nueva geografía del turismo. Barcelona: Ariel, 1997.

VERDÚ, V. El estilo del mundo: la vida en el capitalismo de ficción. Madrid: Anagrama, 2003.

VO, H. V.; SCOTT, M.; COURTNEY, J. F. An empirical comparison of Collective Causal Mapping Approaches. In: NARAYANAN, V. K.; ARMSTRONG, D. J. (Orgs.), Causal Mapping for Research in Information Technology, Hershey, PA: IGI Global, 2005. p. 142-173. 
WARD, J. C.; REINGEN, P. H. Sociocognitive Analysis of Group Decision Making among Consumers. Journal of Consumer Research, v. 17, p. 245-262, 1990.

WEBER, P. S.; MANING, M. R. Cause maps, sensemaking and planning organizational change. Journal of Applied Behavioral Science, v. 37, n. 2, p. 227-251, 2001.

WEICK, K. E. The Social Psycology of Organizing. New York: McGraw-Hill, 1979.

WEICK, K. E.; BOUGON, M. G. Organizations as cognitive maps: Charting ways to success and failure. In: SIMS, H.; GIOIA, D. (Orgs.), The thinking organization: Dynamics of organizational cognition. San Francisco, CA: Jossey-Bass, 1986. p. 102135.

WOODSIDE, A. Training exercise in interpreting causal maps in tourism research. International Journal of Culture, Tourism and Hospitality Research, v. 1, n. 2, p. 175-179, 2007.

Recebido em: 28-08-2012.

Aprovado em: 28-09-2012. 